\title{
GameFlow and Player Experience Measures
}

\author{
An Initial Comparison of Conceptual Constructs \\ Penny Sweetser \\ Australian National University \\ Canberra ACT Australia \\ penny.kyburz@anu.edu.au \\ Daniel Johnson \\ Queensland University of Technology \\ Brisbane QLD Australia \\ dm.johnson@qut.edu.au
}

\begin{abstract}
GameFlow is a widely used model of player enjoyment, which has been applied to designing and evaluating many games and gamelike experiences since its first publication. Although GameFlow has been used as a basis for many surveys, no formal operationalisation has been proposed or validated. This raises the question of whether the development of a GameFlow measure is a worthwhile endeavour. In this paper, we report findings of a study in which GameFlow was used as an evaluation tool for an online strategy game, in conjunction with player experience measures. Expert reviews, using the GameFlow criteria, were conducted by 12 stakeholders (developers, players, researchers, and journalists) in the game Neptune's Pride 2: Triton. Player experience surveys, incorporating the Flow State Scale and Player Experience of Need Satisfaction, were also completed by 351 players. We reflect on the interaction, conceptual constructs, and value of each instrument, and where future development of the GameFlow model should focus.
\end{abstract}

\section{Introduction}

GameFlow [30] is a model of player enjoyment, consisting of 38 criteria derived from games user experience literature and structured into eight elements that conceptually map to Csikszentmihalyi's [6] concept of flow. Since the original publication of the GameFlow model [30], it has seen extensive use throughout the games research and development communities, as well as a number of related areas. The elements and criteria of the GameFlow model have been used in the design and evaluation of a wide range of games and non-game applications, including games for older people [21], disabled people [22], and children [8, 26]; games for education [18, 19], training [2, 10] and rehabilitation [1, 20]; and games for environment [25] and art [13]. The GameFlow model has been used to design and evaluate games for a variety of platforms, including console games [3, 27], mobile games [12, 20, 27], and virtual, augmented, and mixed reality games $[9,13,16,29]$. Several new models have also been derived from the original GameFlow model, including EGameFlow [11], Pervasive GameFlow [15], and MIU-GameFlow [31].

Although GameFlow has been used as an evaluation tool for many different projects and as the foundation for various surveys, there is no validated operationalisation of the model. Rather than attempting to develop a formalized GameFlow measure, in this paper we first seek to investigate the relationship between GameFlow and existing player experience measures, and the potential to use both approaches in tandem. We combined two different approaches to investigate player enjoyment in the online multiplayer game Neptune's Pride 2: Triton. First, expert reviews using the GameFlow criteria were conducted by 12 different stakeholders, including researchers, journalists, players, and the game's developer. Second, quantitative player experience surveys, incorporating the Flow State Scale and Player Experience of Need Satisfaction, were completed by 351 players of the game. Subsequently, we analysed overlaps and divergences between the conceptual constructs of GameFlow and the player experience measures and addressed the different roles each instrument could play in game development. Finally, we concluded that GameFlow provides a unique contribution as a design and evaluation tool for in-depth inquiry, which works alongside validated player experience measures, and that valuable future work lies in further developing the GameFlow model to this purpose. 


\section{GameFlow and Player Experience}

In the original GameFlow paper [30], the key elements of player enjoyment in video games were identified by conducting a comprehensive review of the literature on usability and user experience in games. The result was the identification of eight core elements of player enjoyment in games - concentration, challenge, skills, control, clear goals, feedback, immersion, and social interaction. These core elements were observed to overlap closely with, and were subsequently mapped to, the elements of flow [6]. This structure formed the foundation of a new model of player enjoyment in games, called GameFlow.

The GameFlow model includes a set of heuristics for designing games and performing evaluations via expert reviews. As identified in [27], as well as the player experience literature [4, 5], the process for assessing GameFlow to date has not included player evaluations and no operationalisation has been proposed [7] for converting the GameFlow model into a measure. In order to begin assessing whether the development of a GameFlow measure would be a useful addition to the field, and to better understand how GameFlow fits with existing player experience measures, we selected two player experience measures, the Player Experience of Need Satisfaction [24] and Flow State Scale-2 [14], to use alongside GameFlow. The Player Experience of Need Satisfaction (PENS) measure is based on self-determination theory [23], which is a theory of motivation that includes intrinsic and extrinsic motives. The Flow State Scale-2 (FSS-2) is a measure designed to assess flow experiences in physical activity. The GameFlow elements conceptually overlap with the constructs of each measure as shown in Table 1.

Table 1: Conceptual Overlap between GameFlow and PENS and FSS-2 constructs.

\begin{tabular}{|c|c|c|}
\hline GameFlow & PENS & FSS-2 \\
\hline Concentration & & Concentration \\
\hline Challenge & Competence & Challenge-Skill \\
\hline Player Skills & $\begin{array}{l}\text { Intuitive } \\
\text { Controls }\end{array}$ & \\
\hline Control & $\begin{array}{l}\text { Autonomy, } \\
\text { Intuitive } \\
\text { Controls }\end{array}$ & Control \\
\hline Clear Goals & & Clear Goals \\
\hline Feedback & & Unambiguous Feedback \\
\hline Immersion & Presence & $\begin{array}{l}\text { Action Awareness, } \\
\text { Self-Consciousness, } \\
\text { Transformation of Time }\end{array}$ \\
\hline \multirow[t]{2}{*}{$\begin{array}{l}\text { Social } \\
\text { Interaction }\end{array}$} & Relatedness & \\
\hline & & Autotelic Experience \\
\hline
\end{tabular}

The PENS is a 21-item scale that measures player experience across five factors, which are rated by respondents on a 7-point Likert scale, ranging from (1) do not agree to (7) strongly agree:

- $\quad$ Competence (three items assessing players' perception that the game was optimally challenging). There is strong conceptual overlap between this scale and the GameFlow element of Challenge.

- Autonomy (three items assessing players' experienced choice, freedom, and activities that interested them). There is strong conceptual overlap between this scale and the GameFlow element of Control.

- $\quad$ Relatedness (three items assessing how connected players felt to other players in the game). There is conceptual overlap between this scale and the GameFlow element of Social Interaction.

- $\quad$ Presence (nine items assessing players' sense of immersion in the gaming environment). There is strong conceptual overlap between this scale and the GameFlow element of Immersion.

- Intuitive Controls (three items assessing the interface that controlled participants' actions in the game). There is conceptual overlap between this scale and the GameFlow elements of Control and Player Skills.

The FSS-2 is a 36-item scale that measures flow across nine factors: Challenge-Skill Balance, Action Awareness, Clear Goals, Unambiguous Feedback, Concentration, Sense of Control, Self-Consciousness, and Transformation of Time. Responses are rated on a 5-point Likert scale ranging from (1) strongly disagree to (5) strongly agree. The FSS-2 scales conceptually overlap with GameFlow elements as shown in Table 1. 


\section{Method}

Our method for this research involved combining two different approaches to evaluating the online multiplayer strategy game, Neptune's Pride 2: Triton (NP2). The first approach involved 12 different stakeholders in NP2, including expert players (N=7), journalists $(\mathrm{N}=2)$, researchers $(\mathrm{N}=2)$, and the game's developer $(\mathrm{N}=1)$, conducting expert reviews using the GameFlow criteria. The second approach involved 351 players of NP2 completing the player experience measures, PENS and FSS-2. Subsequently, ratings, correlations, and resulting overlaps were calculated and analysed.

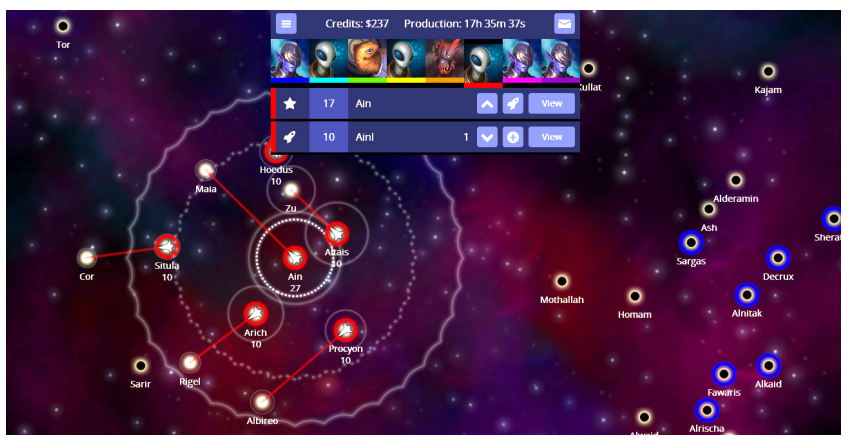

Figure 1: Neptune's Pride 2: Triton (np.ironhelmet.com) is a browser-based 4x space conquest game.

\subsection{Neptune's Pride II: Triton}

Neptune's Pride (NP) is an abstract science fiction strategy game, belonging to the 4x (explore, expand, exploit, exterminate) genre of games. NP is a slow real-time game, which plays out over weeks or months. NP was originally released in 2010 and won honourable mention in the 2011 Independent Games Festival Seamus McNally Grand Prize category at the Game Developers' Conference. It was also awarded PC Gamer UK's Webgame of the Year in 2011. In 2013, Iron Helmet Games won a grant from Screen Australia to develop a new version of NP. NP2 is an update and expansion to the original game (see Figure 1). The main changes were technology based, moving from Adobe Flash to native browser technologies (HTML and Javascript), so that the game would accommodate mobile devices. There were also changes to mechanics, art, and tuning to improve the overall game experience.

\subsection{GameFlow Reviews}

In our current study, we applied the GameFlow criteria in its original form, as in $[27,28,30]$. Reviewers were selected based on their familiarity with the game and their expertise in their relevant area, including journalists who had reviewed the original game, expert players, and researchers with expertise in games design and player experience. Most of the expert players (71\%) had completed over 20 games of NP and all had completed at least one game each of NP and the beta version of NP2. All reviewers completed at least one game of NP2 beta prior to performing their review. It is important to note that a single game of NP/2 often takes weeks to play out.

The reviewers completed the GameFlow review via a Google Form. For each GameFlow criterion, each reviewer assigned a numerical value between one and five, indicating the extent to which the game supports that criterion. Values from one to five

Table 2: FSS-2 correlation to PENS. Significant correlations included, with ${ }^{* *} \mathbf{p}<.01,{ }^{*} \mathbf{p}<.05$.

\begin{tabular}{l|lllll} 
& $\begin{array}{l}\text { PENS } \\
\text { Competence }\end{array}$ & $\begin{array}{l}\text { PENS } \\
\text { Autonomy }\end{array}$ & $\begin{array}{l}\text { PENS } \\
\text { Relatedness }\end{array}$ & $\begin{array}{l}\text { PENS } \\
\text { Presence }\end{array}$ & $\begin{array}{l}\text { PENS } \\
\text { Int Controls }\end{array}$ \\
\hline FSS-2 Challenge Skill & $.748^{* *}$ & $.516^{* *}$ & $.242^{* *}$ & $.287^{* *}$ & $.337^{* *}$ \\
FSS-2 Action Awareness & & & & & $.140^{*}$ \\
FSS-2 Clear Goals & $.579^{* *}$ & $.376^{* *}$ & & $.397^{* *}$ \\
FSS-2 Unambiguous Feedback & $.614^{* *}$ & $.395^{* *}$ & $.141^{*}$ & $.209^{* *}$ & $.376^{* *}$ \\
FSS-2 Concentration & $.450^{* *}$ & $.416^{* *}$ & $.220^{* *}$ & $.337^{* *}$ & $.279^{* *}$ \\
FSS-2 Sense of Control & $.590^{* *}$ & $.552^{* *}$ & $.146^{* *}$ & $.245^{* *}$ & $.382^{* *}$ \\
FSS-2 Self-Consciousness & $.135^{* *}$ & & $-.162^{* *}$ & & $.400^{* *}$ \\
FSS-2 Transformation of Time & $.177^{* *}$ & $.186^{* *}$ & $.214^{* *}$ & $.363^{* *}$ & $.371^{* *}$
\end{tabular}


indicate "not at all," "below average," "average," "above average," and "well done," respectively. These values were combined into mean scores for each GameFlow element, as well as an overall GameFlow score (the mean of all GameFlow element scores) for the game. The reviewers were also asked to provide written qualitative comments to explain their given rating for each criterion.

\subsection{Player Experience Measures}

In addition to expert reviews using the GameFlow model, we collected survey responses from players of the online multiplayer strategy game, NP2. Participants were recruited via a notice on the game's online forum. A total of 441 people started the survey, but a large number $(n=90)$ only provided data related to demographics and play habits (exiting the survey before providing any responses for the FSS-2 or PENS). The current paper focuses on the remaining 351 participants (who provided responses to our substantive measures). A total of 332 males and 17 females completed the survey with an age range of 17 to 47 years (mean age of 27.5 years, standard deviation of 6.3 years). All participants had completed at least one game of NP2 or NP1. On average participants had completed 2.05 games of NP2 (standard deviation 2.37, range 0-28) and 3.49 games of NP1 (standard deviation 9.39, range 096). Participants completed the PENS and FSS-2 questionnaires via an online survey.

\section{Results}

For the GameFlow elements, NP2 received relatively high ratings (above 3.5 out of 5) in Concentration (Mean=4.0), Control $(M e a n=4.0)$, Clear Goals (Mean=4.1), Social Interaction (Mean=4.1), and Feedback (Mean=3.8). Lower ratings were achieved for Challenge (Mean=2.6), Player Skills (Mean=3.4), and Immersion (Mean=3.4). For the PENS, NP2 achieved a relatively high score (above 5 out of 7) for Competence (Mean=5.39, $S D=1.045)$, Autonomy (Mean=5.33, $S D=1.134$ ), and Intuitive Controls (Mean=5.45, $S D=1.194$ ) and a lower score for Presence (Mean=3.71, $S D=1.133$ ). For the FSS-2, NP2 achieved a relatively high score (above 3.5 out of 5) for Concentration (Mean=3.61, $S D=0.772)$, Challenge Skill Balance (Mean=3.9, $S D=0.649)$, Clear Goals $(M e a n=3.95, S D=0.76)$, Unambiguous Feedback (Mean=3.93, $S D=.0712)$, Sense of Control (Mean=4.0, $S D=0.671)$, and Autotelic Experience $(M e a n=3.99$, $S D=0.683$ ) and achieved lower scores for Action Awareness (Mean=2.6, $S D=0.766)$, Self-Consciousness $(M e a n=2.9, S D=1.062)$, and Transformation of Time (Mean=2.87, $S D=0.932$ ). PENS and FSS-2 correlations are presented in Table 2. PENS Competence was found to have strong correlations with FSS-2 Challenge Skill, Clear Goals, Unambiguous Feedback, Sense of Control, and Autotelic Experience. PENS Autonomy was found to have strong correlations with FSS-2 Challenge Skill, Sense of Control, and Autotelic Experience.

\section{Discussion}

The results helped us to gain insight into how the game we reviewed with the GameFlow model performed on validated measures of player experience and to compare the results with our GameFlow review results. If we revisit the earlier conceptual mapping between GameFlow, PENS, and FSS-2, we can see where this mapping was supported or contrasted by the results.

- $\quad$ Both GameFlow Concentration and FSS-2 Concentration received relatively high ratings.

- Both PENS Competence and FSS-2 Challenge-Skill Balance received relatively high ratings, whereas GameFlow Challenge received a relatively low score.

- $\quad$ All of GameFlow Control, PENS Autonomy and Intuitive Controls, and FSS-2 Control received relatively high ratings.

- Both GameFlow Clear Goals and FSS-2 Clear Goals received relatively high ratings.

- Both GameFlow Feedback and FSS-2 Unambiguous Feedback received relatively high ratings.

- Both PENS Presence and FSS-2 Action Awareness, Self-Consciousness, and Transformation of Time received relatively low scores, whereas GameFlow Immersion received a relatively low score.

- GameFlow Social Interaction received a relatively high score, whereas PENS Relatedness received a relatively low rating.

The discrepancy between FSS-2 Challenge-Skill and GameFlow Challenge could reflect difficulties associated with using the FSS2 in relation to videogames. Previous research [22] has suggested that the Challenge-Skill balance subscale (of the FSS-2) may erroneously suggest relatively high levels of flow in situations where respondents feel their skills outstrip the present challenge (i.e., situations in which they feel competent, regardless of a particularly good match between skill and challenge). Our results suggest that the GameFlow expert review for Challenge might provide a more nuanced view of this construct (i.e., in situations where players have enough skill to play the game but are not being challenged sufficiently to increase the likelihood of flow, a relatively low rating is shown). Further research is needed in order to confirm this possibility.

In addition, the GameFlow Social Interaction component might better be viewed as a measure of whether a particular game has the structure (or potential) to support interactions between players, while the relatedness subscale of the PENS, in contrast, indicates 
whether a particular game has been successful in facilitating positive connections. This raises a broader question of whether each element and criteria are evaluating the player experience or the game (or potential player experience).

PENS and FSS-2 correlation analyses additionally revealed relatively strong correlations (over 0.5) between:

- $\quad$ PENS Competence and FSS-2 Challenge-Skill Balance, Clear Goals, Unambiguous Feedback, Sense of Control, and Autotelic Experience.

- $\quad$ PENS Autonomy and FSS-2 Challenge Skill, Sense of Control, and Autotelic Experience.

The results support the conceptual overlap between GameFlow, PENS, and FSS-2, but demonstrate that the subscales are also cross-sectional and not aligned. PENS Competence and Autonomy have strong correlations across multiple subscales of the FSS-2. Social experience is not represented in the FSS-2, which results in relatively weak (or non-significant) correlations with the PENS Relatedness subscale. PENS Presence and Intuitive Controls also show relatively weak (or non-significant) correlations with FSS-2 subscales. Similarly, FSS-2 Concentration, Action Awareness, Self-Consciousness, and Transformation of Time showed nonsignificant or relatively weak correlations with PENS subscales. Interestingly, the PENS and FSS-2 subscales related to presence, immersion, and the characteristic flow experience did not have strong correlations and neither did they reflect the scores returned by the GameFlow immersion criteria. This could reflect the unique player experience offered by NP2, but also illustrates the difficulty in reliably measuring the experiences of flow, presence, and immersion.

\section{Conclusion}

Employing the PENS and FSS-2 alongside the GameFlow model provided interesting insight into the player experience from the perspective of each measure. Our study highlighted differences and similarities between the different measures. Some of which confirmed our hypothesised conceptual overlaps, and some which raised further questions. Each measure provides a rating for the extent to which a game is providing an enjoyable player experience. However, as we have seen in this study, ratings alone are unlikely to provide a complete understanding of why and how a game is providing an enjoyable experience or to provide all available insights to a developer who is trying to improve their game.

We propose that there are already an extensive, and growing, number of player experience measures available for researchers and that a key opportunity for use and future development of the GameFlow model lies in supporting developers and researchers to inquire into how and why a game is or isn't providing, or affording, an enjoyable experience, via heuristics. Heuristics have the twin benefits of drawing on the expertise of the person applying them and have the potential to provide greater depth of insight than numerical ratings. We propose future research further explore the application and refinement of the GameFlow heuristics to different genres of games and across a variety of experts.

\section{REFERENCES}

[1] Per Backlund, Anna-Sofia Alklind Taylor, Henrik Engström, Mikael Johannesson, Mikael Lebram, Angelique Slijper, Karin Svensson, Jesper Poucette, and Katharina Stibrant Sunnerhagen. 2013. Games on prescription! Evaluation of the Elinor console for home-based stroke rehabilitation. In Transactions on edutainment IX, 49-64. https://doi.org/10.1007/978-3-642-37042-7_3.

[2] Sayli Bhide. 2017. Fire Safety and Emergency Evacuation Training for Occupants of Building Using 3D Virtual Simulation. Ph.D. Dissertation. University of Central Florida, Orlando, FL.

[3] Shaw Bronner, Russell Pinsker, and J. Adam Noah. 2013. Energy cost and game flow of 5 exer-games in trained players. American journal of health behavior 37, 3: 369380. https://doi.org/10.5993/AJHB.37.3.10.

[4] Dustin Chertoff. 2009. Exploring additional factors of presence. Ph.D. Dissertation. University of Central Florida, Orlando, FL.

[5] Kimberly Chu, Chui Yin Wong, and Chee Weng Khong. 2011. Methodologies for evaluating player experience in game play. In International Conference on HumanComputer Interaction, 118-122. https://doi.org/10.1007/978-3-642-22098-2_24.

[6] Mihaly Csikszentmihalyi. 1991. Flow: The Psychology of Optimal Experience. Harper Perennial, New York.

[7] Frederik De Grove, Jan Van Looy, Cédric Courtois, and Lieven De Marez. 2010. I play, therefore I learn? Measuring the Evolution of Perceived Learning and Game Experience in the Design Flow of a Serious Game. Meaningful Play. Michigan State University, Michigan, 1-32.

[8] Iván Durango, Alicia Carrascosa, Jose A. Gallud, and Victor MR Penichet. 2018. Interactive fruit panel (IFP): a tangible serious game for children with special needs to learn an alternative communication system. Universal Access in the Information Society 17, 1: 51-65. https://doi.org/10.1007/s10209-016-0517-5.

[9] Samantha Finkelstein, Andrea Nickel, Zachary Lipps, Tiffany Barnes, Zachary Wartell, and Evan A. Suma. 2011. Astrojumper: Motivating exercise with an immersive virtual reality exergame. Presence: Teleoperators and Virtual Environments 2011 20, 1: 78-92. http://dx.doi.org/10.1162/pres_a_00036.

[10] Nicolai Foldager, Hans Hansen, Mikkel Skovsmose Tewes, and Thomas Bjørner. 2016 Designing an Engaging and Informative Application About First Aid: Gamification and Humor as Design Elements in a Serious Game. In International Conference on Smart Objects and Technologies for Social Good, 78-87. https://doi.org/10.1007/9783-319-61949-1_9.

[11] Fong-Ling Fu, Rong-Chang Su, and Sheng-Chin Yu. 2009. EGameFlow: A scale to measure learners' enjoyment of e-learning games. Comput. Educ. 52, 1 (January 2009), 101-112. http://dx.doi.org/10.1016/j.compedu.2008.07.004.

[12] Andreas Gustafsson and Jesper Vallett. 2018. Context-Aware Procedural Content Generation with Player Modelling in Mobile Action Role Playing Game. Bachelor thesis, Malmö University, Malmö, Sweden.

[13] Meng-Hsuan Huang and Saiau-Yue Tsau. 2018. A Flow Experience Analysis on the Virtual Reality Artwork: La Camera Insabbiata. In Proceedings of the International Conference on Machine Vision and Applications, 51-55.

[14] Susan A. Jackson and Robert C. Eklund. 2002. Assessing flow in physical activity: The flow state scale-2 and dispositional flow scale-2. Journal of Sport and Exercise Psychology 24.2: 133-150. 
[15] Kalle Jegers. 2009. Elaborating eight elements of fun: Supporting design of pervasive player enjoyment. Comput. Entertain. 7, 2, Article 25 (June 2009), 22 pages. https://doi.org/10.1145/1541895.1541905.

[16] Eng Tat Khoo, Adrian David Cheok, Ta Huynh Nguyen, and Zhigeng Pan. 2008. Age invaders: social and physical inter-generational mixed reality family entertainment. Virtual Real.12, 1 (March 2008), 3-16. https://doi.org/10.1007/s10055-008-0083-0.

[17] Madison Klarkowski, Daniel Johnson, Peta Wyeth, Simon Smith, and Cody Phillips. 2015. Operationalising and Measuring Flow in Video Games. In Proceedings of the Annual Meeting of the Australian Special Interest Group for Computer Human Interaction (OzCHI '15), 114-118. https://doi.org/10.1145/2838739.2838826.

[18] Herbert Lange and Peter Ljunglöf. 2018. MULLE: A grammar-based Latin language learning tool to supplement the classroom setting. In Proceedings of the 5th Workshop on Natural Language Processing Techniques for Educational Applications, 108-112.

[19] Richard WC Lui and Cheuk Hang Au. 2017. Designing simulation games for information systems education-A case study in teaching for digital marketing. In 2017 IEEE 6th International Conference on Teaching, Assessment, and Learning for Engineering (TALE), 290-295. https://doi.org/10.1109/TALE. 2017.8252349.

[20] Charlotte Magnusson, Kirsten Rassmus-Gröhn, Bitte Rydeman, and Héctor Caltenco. 2018. Walk after stroke: initial development of a step counting game for stroke survivors. In 20th International Conference on Human-Computer Interaction with Mobile Devices and Services Adjunct (MobileHCI'18), $237-237$. https://doi.org/10.1145/3236112.3236145.

[21] Wytske Meekes and Emma Kate Stanmore. 2017. Motivational determinants of exergame participation for older people in assisted living facilities: mixed-methods study. Journal of medical Internet research 19, 7: e238. http://doi.org/10.2196/jmir.6841.

[22] Kyle Rector, Roger Vilardaga, Leo Lansky, Kellie Lu, Cynthia L. Bennett, Richard E. Ladner, and Julie A. Kientz. 2017. Design and Real-World Evaluation of Eyes-Free Yoga: An Exergame for Blind and Low-Vision Exercise. ACM Trans. Access. Comput. 9, 4, Article 12 (April 2017), 25 pages. https://doi.org/10.1145/3022729.

[23] Richard M. Ryan and Edward L. Deci. 2000. Self-determination theory and the facilitation of intrinsic motivation, social development, and well-being. American psychologist 55, 1: 68.

[24] Richard M. Ryan, C. Scott Rigby, and Andrew Przybylski. 2006. The motivational pull of video games: A self-determination theory approach. Motivation and emotion 30, 4: 344-360.

[25] Sonja Schaal, Siegmar Otto, Steffen Schaal, and Armin Lude. 2018. Game-related enjoyment or personal pre-requisites-which is the crucial factor when using geogames to encourage adolescents to value local biodiversity. International Journal of Science Education, Part B 8, 3: 213-226.

[26] Marijn Speelman and Ben Kröse. 2008. Virtual mirror gaming in libraries. In ECAG 2008 Workshop Facial and Bodily Expressions for Control and Adaptation of Games, 37.

[27] Penelope Sweetser, Daniel Johnson, Peta Wyeth, Aiman Anwar, Yan Meng, and Anne Ozdowska. 2017. GameFlow in Different Game Genres and Platforms. Comput. Entertain. 15, 3, Article 1 (April 2017), 24 pages. https://doi.org/10.1145/3034780.

[28] Penny Sweetser and Daniel Johnson. 2019. Evaluating the GameFlow Model with Different Stakeholders. In Proceedings of the 2019 Annual Symposium on ComputerHuman Interaction in Play Companion Extended Abstracts (CHI PLAY '19 Extended Abstracts). DOI: https://doi.org/10.1145/3341215.3356286

[29] Penny Sweetser, Zane Rogalewicz and Qingyang Li. 2019. Understanding Enjoyment in VR Games with GameFlow. In Proceedings of VRST '19: 25th ACM Symposium on Virtual Reality Software and Technology (VRST '19), 2 pages. DOI: https://doi.org/10.1145/3359996.3364800

[30] Penelope Sweetser and Peta Wyeth. 2005. GameFlow: a model for evaluating player enjoyment in games. Comput. Entertain. 3, 3 (July 2005), 3-3. https://doi.org/10.1145/1077246.1077253.

[31] Nurul Hidayah Mat Zain, Azizah Jaafar, and Fariza Hanis Abdul Razak. 2016. Enjoyable game design: validation of motor-impaired user GameFlow model. International Journal of Computer Theory and Engineering 8, 2: 116. 